\title{
Plasmonic versus all-dielectric nanoantennas for refractometric sensing: a direct comparison.
}

Noemi Bosio, 1, Hana Šípová-Jungová, ${ }^{1}$ Nils Odebo Länk, ${ }^{1}$ Tomasz J. Antosiewicz, 1,2

Ruggero Verre, ${ }^{1 \#}$ Mikael Käl/1\#

1Department of Physics, Chalmers University of Technology, 41296 Göteborg,

\section{Sweden.}

2Faculty of Physics, University of Warsaw, Pasteura 5, 02-093 Warsaw, Poland.

*Corresponding authors: ruggero.verre@chalmers.se; mikael.kall@chalmers.se
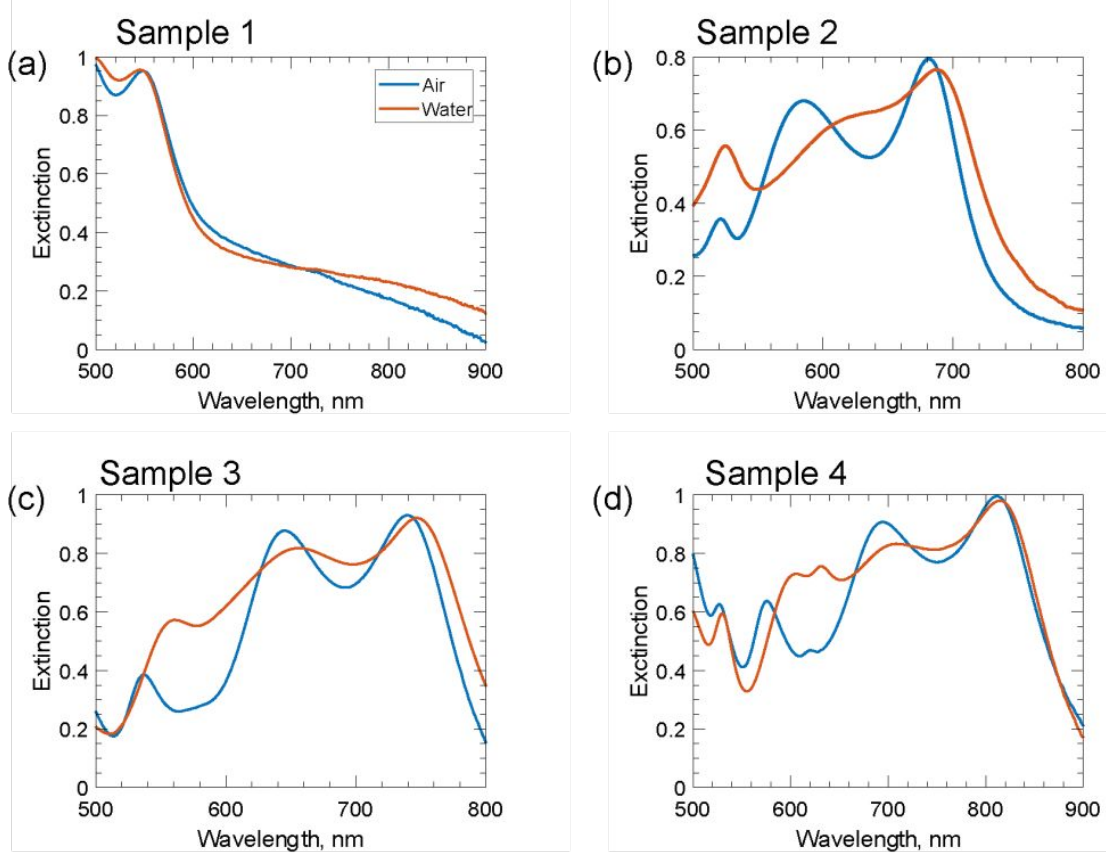
Figure S1. Extinction spectra of different samples made of Si nanodisk arrays with different in-plane diameter and height measured in air (blue lines) and water (orange lines). The height and diameter for the various samples are indicated in Table 1.
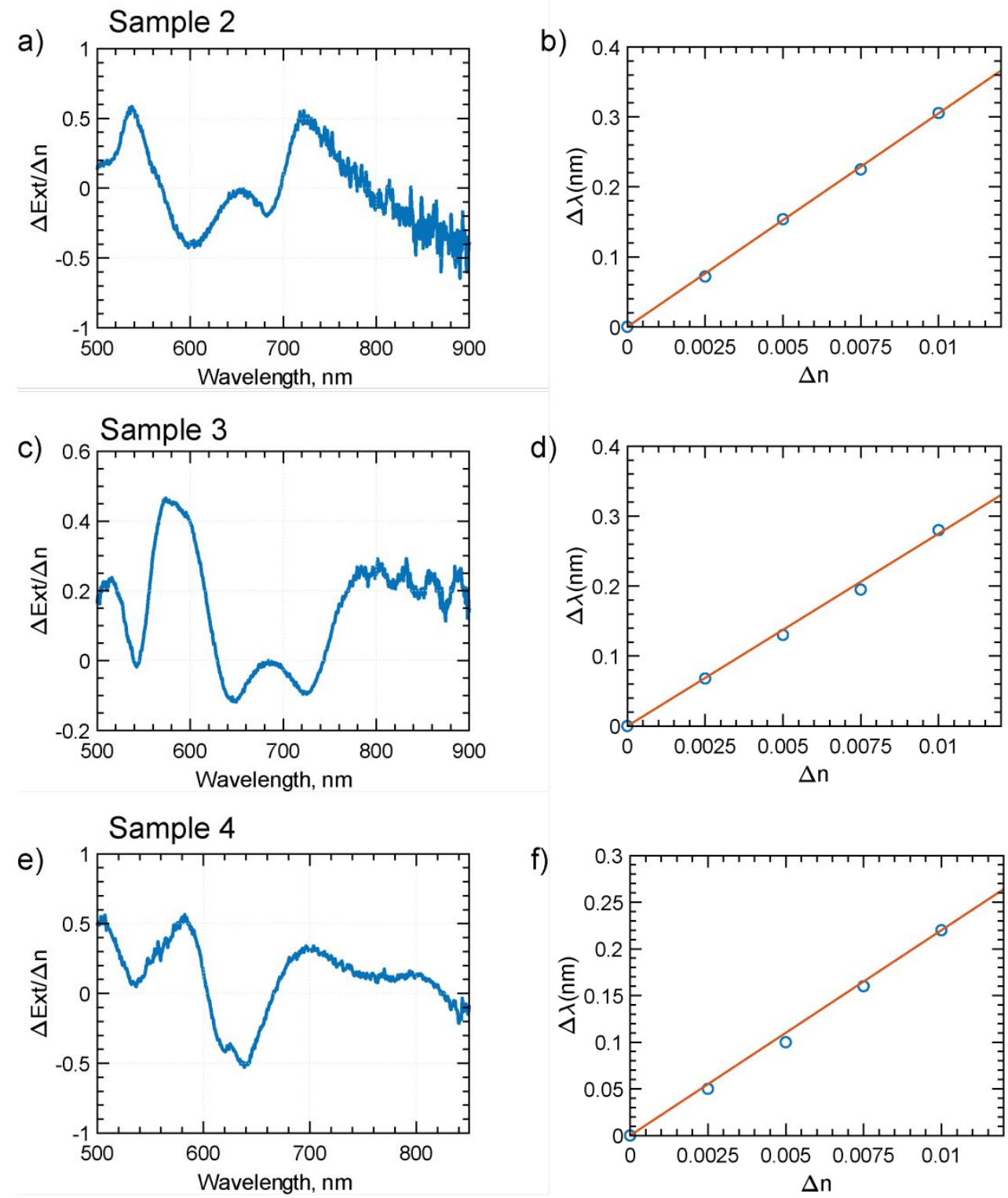

Figure S2. Bulk refractometric sensing for different Si nanoparticle arrays. (a-c-e) Change in extinction intensity per refractive index unit as a function of wavelength for different $\mathrm{Si}$ samples and (b-d-f) change of wavelength position for the magnetic dipolar resonance as a function of the refractive index of the surrounding medium 


\begin{tabular}{|c|c|c|c|}
\hline Samples & Sensitivity [nm/RIU] & Diameter & Height \\
\hline Sample 1 & NA & 100 & 144 \\
\hline Sample 2 & $30.5 \pm 1.2$ & 150 & 144 \\
\hline Sample 3 & $27.5 \pm 3.0$ & 150 & 200 \\
\hline Sample 4 & $22 \pm 2.0$ & 170 & 200 \\
\hline Au & $216 \pm 30$ & 150 & 30 \\
\hline
\end{tabular}

Table S1. Sensitivity defined as change in the magnetic dipole resonance position per refractive index for different samples discussed in the article. The respective sizes (diameter and height) is also indicated for reference. Note that for sample 1 , as the changes from air to water were minimal (cfr. Fig.S1), bulk refractive index sensing was not performed.
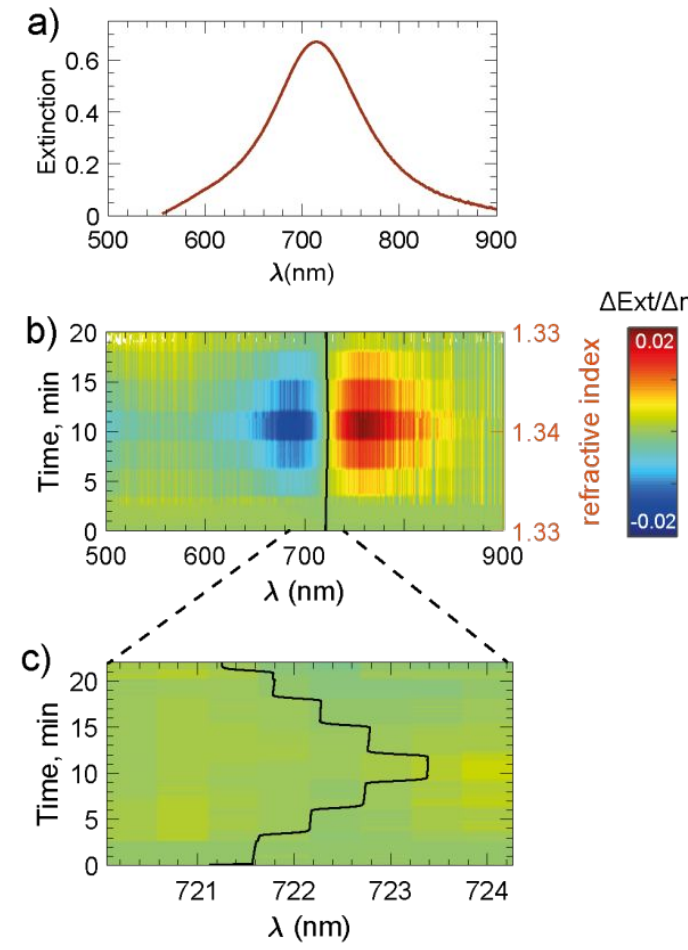

Figure S3. Au bulk refractrometric sensing maps. (a) Extinction spectra for Au sample discussed in the main text. (b) Difference in extinction per refractive index unit change, when the 
surrounding medium is changed from water $(\mathrm{RI}=1.33, \mathrm{t}=0$ minutes) to $10 \%$ ethylene glycol (RI $=1.34, t=10$ minutes) as a function of time. The black line traces the centroid wavelength position of the plasmonic resonance as a function of time. (c) The same data as $b$ in a narrower wavelength region, to outline the change in wavelength position in different refractive index media.
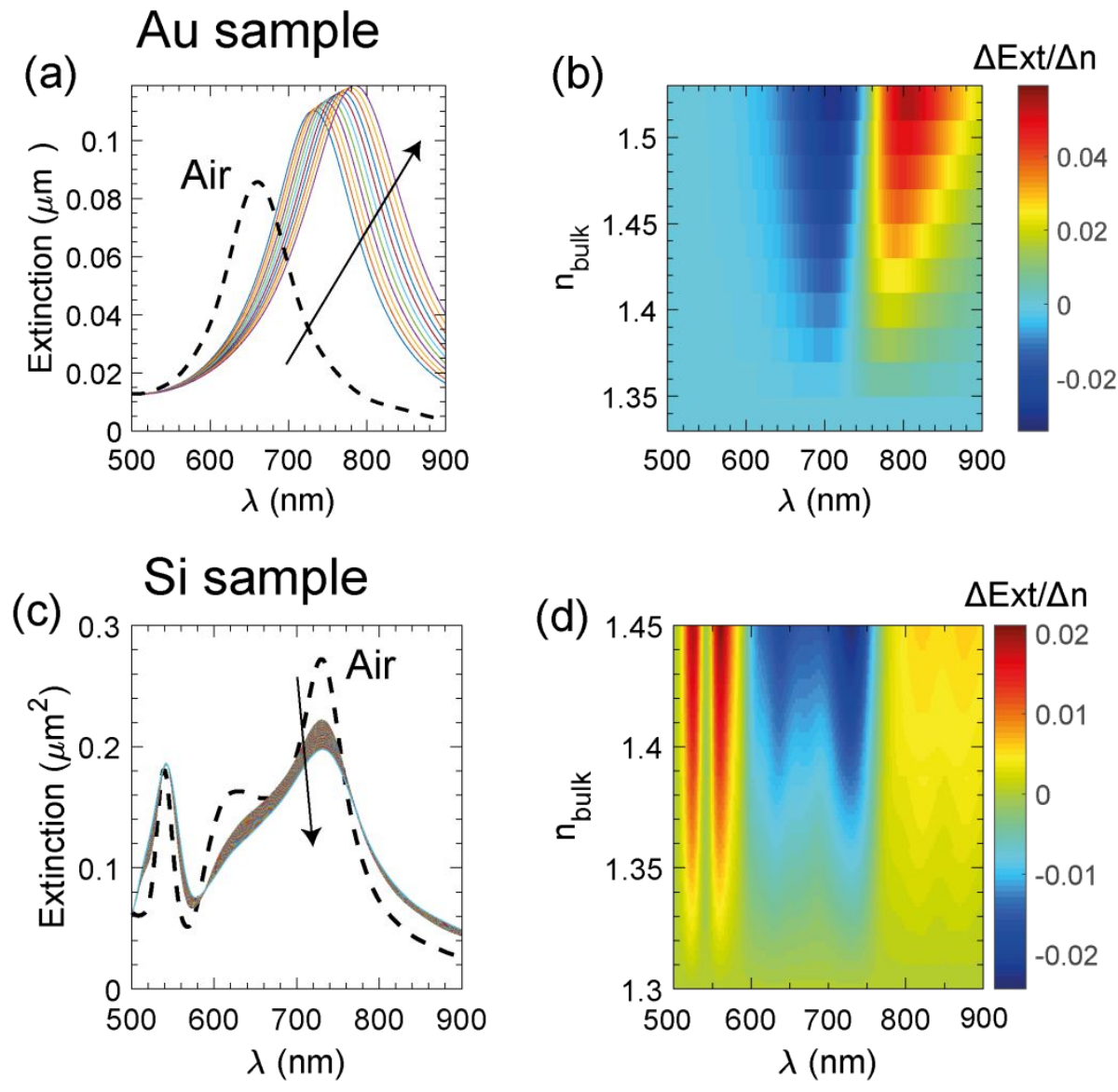

Figure S4. Simulated bulk sensitivity for single Si and Au nanodisks discussed in the text. (a-c) Extinction spectra in air (dashed line) and in different refractive indexes from 1.33 to .144. The arrow indicate spectral changes when the refractive index is increased. (b-d) Simulated Difference in extinction cross section per refractive index unit change as a function of the wavelength, when the surrounding medium is changed from $n=1.33$ to $n=1.45$. 

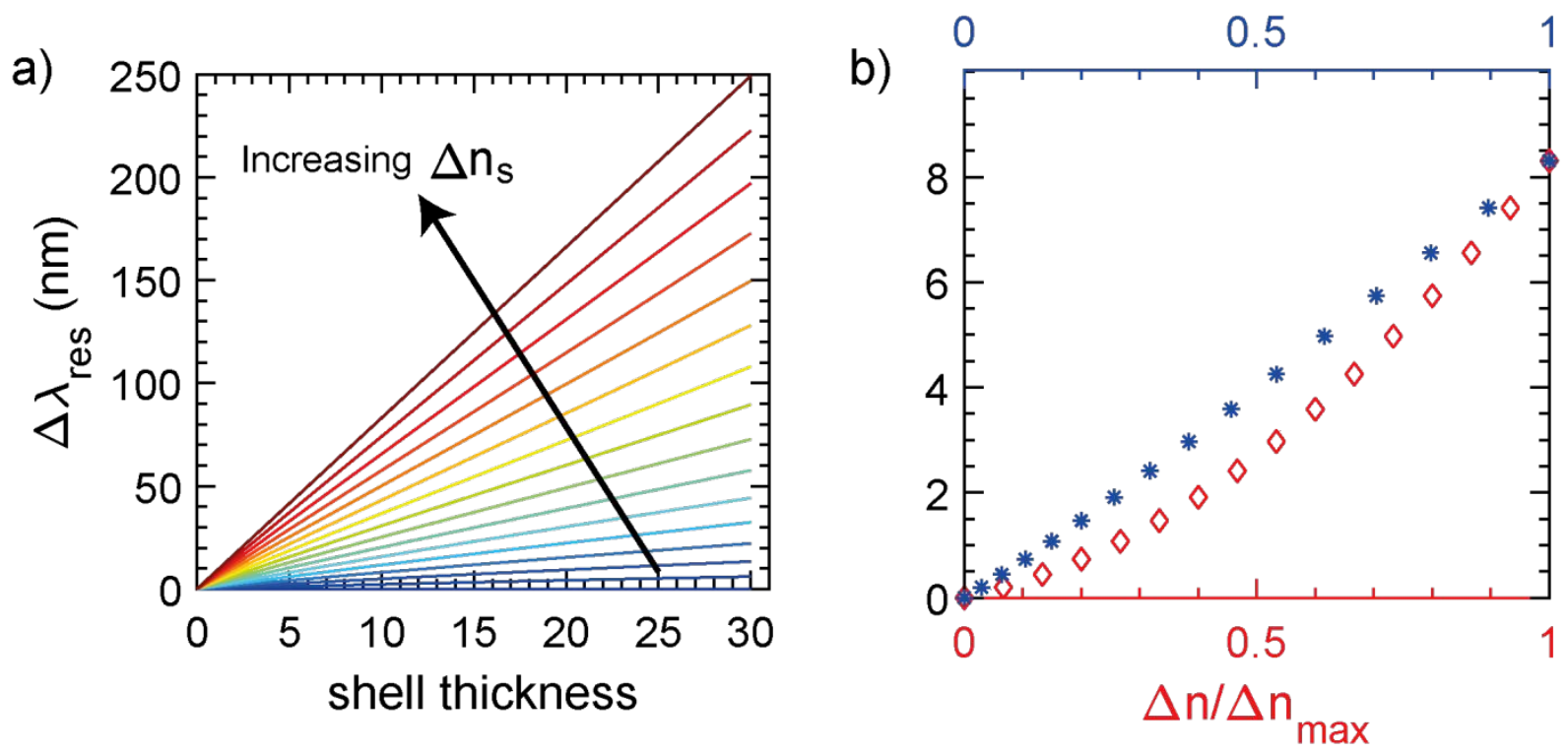

Figure S5. Mie theory calculations of a core-shell sphere made of poly-Si and coated by a thin dielectric shell. The magnetic resonance wavelength shift is linear as a function of the shell thickness for different shell refractive indexes $\Delta n_{S}$. (b) The sensitivity of the magnetic resonance shift per normalized change of refractive index $\Delta n_{S}$ (bottom) and $\Delta \varepsilon_{S}$ (top) indicates that the resonance position shifts linearly with $\Delta \varepsilon_{S}$. 